\title{
Prevalência e caraterísticas das Quedas de idosos institucionalizados*
}

\author{
Prevalence and features of falls of institutionalized elders \\ Prevalencia y características de las caídas de ancianos institucionalizados
}

Denise Cristina de Oliveira Ferreira', Aparecida Yoshie Yoshitome'

'Universidade Federal de São Paulo. Departamento de Enfermagem. São Paulo, SP

Submissão: 25/05/2010

Aprovação: $27 / 07 / 2010$

\section{RESUMO}

Trata-se de estudo retrospectivo para verificar prevalência de Quedas em residentes de Instituição de Longa Permanência para Idosos, em São Paulo, caracterizar os idosos caidores e descrever os eventos. Analisamos 121 prontuários e 87 relatórios de Quedas, entre agosto de 2006 e agosto de 2007. Encontramos II4 Quedas sofridas por 45 idosos, prevalência de 37,2\%. Dentre os caidores, a maioria era mulher, média de idade 83,75 anos. Encontramos Quedas recorrentes, a maioria se deu da própria altura, no Quarto do idoso, gerando como conseQuência principal hematomas. Os residentes apresentavam diversos diagnósticos médicos e polifarmácia. Destacamos a necessidade da implementação de protocolos para prevenção de Quedas, em vista da magnitude do problema e repercussões para capacidade funcional do idoso institucionalizado.

Descritores: Idoso; Instituição de longa permanência para idosos; Acidentes por Quedas; Enfermagem geriátrica.

\section{ABSTRACT}

This retrospective study seeks to check the prevalence of falls in older people from a long-term care institution for elderly people, in São Paulo, to describe the fallers and the events. We analyzed 121 medical records and 87 fall reports, between August 2006 and August 2007. There were 114 falls suffered by 45 ancians, a prevalence of $37,2 \%$. The majority of fallers are women, average age 83,75 years. We found recurrent falls, various diagnoses and polipharmacy. The majority occurred in own height, in their bedroom, producing mainly hematomas. There is a need of implementation of fall preventive guidelines, due to the importance of this issue and its repercussions to the functional status of the institutionalized elderly.

Key words: Aged; Homes for the aged; Accidental falls; Geriatric nursing.

\section{RESUMEN}

Estudio descriptivo retrospectivo con objetivo de verificar la prevalencia de las caídas en adultos mayores residentes en un hogar para ancianos; describir los ancianos y las caídas. Analizamos 121 prontuarios y 87 informes de caídas, entre agosto de 2006 y agosto de 2007. Fueron encontradas 114 caídas sufridas por 45 ancianos, una prevalencia de $37,2 \%$. La mayoría de los que cayeron eran mujeres, con edad promedia de 83,75 años. Fueron encontradas caídas repetidas, gran parte ocurrieron de la propia altura, en la habitación, generando hematomas. Los ancianos presentaban vários diagnósticos médicos y polifarmacia.. Apuntamos la necesidad de implantación de la prevención de caídas, debido a la importancia del problema y sus repercusiones para la capacidad funcional del anciano institucionalizado.

Descriptores: Anciano; Hogares para ancianos; Accidentes por caídas; Enfermería geriátrica.

\section{*Trabalho de conclusão de curso para obtenção do título de Enfermeira pela Universidade Federal de São Paulo (UNIFESP), em 2008.}

AUTOR CORRESPONDENTE Denise Cristina de Oliveira Ferreira. Rua Moinho Velho 83I, apto 33. CEP 02929-000. São Paulo, SP.

E-mail: decris.ferreira@gmail.com 


\section{INTRODUÇÃO}

O envelhecimento da população é um fenômeno de amplitude mundial. A Organização Mundial de Saúde (OMS) prevê Que, em 2025, existirão I,2 bilhões de pessoas com mais de 60 anos, sendo Que as pessoas com 80 anos ou mais constituem o segmento populacional Que mais cresce ${ }^{(1)}$. No Brasil, a previsão é de Que, em 2020, existirão 30,8 milhões de idosos, ou seja, $14,2 \%$ de todos os brasileiros. O Brasil já é considerado o sexto país do mundo em taxa de envelhecimento populacional ${ }^{(2)}$.

Esta transição demográfica repercute na saúde por meio de mudanças no perfil de morbidade e mortalidade da população, traduzindo a necessidade de reorganizar os modelos assistenciais ${ }^{(3)}$. Assim, o atendimento adequado à saúde do idoso deve ser voltado para a prevenção e identificação de sinais e sintomas característicos do envelhecimento com fragilidade, um contínuo e complexo processo envolvendo a interação de fatores biológicos, psicológicos e sociais, culminando num estado favorável à ocorrência de condições geradoras de dependência e institucionalização, como alterações cognitivas, incontinência urinária, instabilidade da marcha e Quedas ${ }^{(4)}$.

As Quedas podem ser definidas como eventos não intencionais Que resultam na mudança de posição inesperada do indivíduo para um nível inferior à posição inicial, com incapacidade de correção em tempo hábil ${ }^{(5)}$. Esses eventos resultam da interação de diversos fatores de risco e múltiplas causas, sendo, por isso, considerados eventos multifatoriais e heterogêneos.

As Quedas são problemas frequentes na população idosa. No Brasil, cerca de $29 \%$ dos idosos caem ao menos uma vez ao ano e $13 \%$ caem de forma recorrente ${ }^{(5)}$. Estes eventos têm impacto importante na mortalidade de idosos. Segundo dados preliminares do Departamento de Informática do SUS (DATASUS), no ano de 2008 ocorreram 5.142 mortes de pessoas com 60 anos ou mais, no país, em decorrência de Quedas, ocupando o segundo lugar na mortalidade por causas externas, com $25,3 \%{ }^{(6)}$.

Além da alta mortalidade, devemos considerar outras conseQuências para a saúde e a Qualidade de vida dos idosos como declínio da capacidade funcional, limitação na realização de atividade física, diminuição da mobilidade, receio de sofrer novas Quedas, isolamento social, perda da autonomia e da independência para execução das atividades de vida diária. Esse Quadro é responsável pela necessidade de institucionalização do idoso frágil ${ }^{(5)}$.

Entende-se por institucionalização o atendimento integral, em regime de internato, às pessoas de 60 anos ou mais, dependentes ou independentes, sem vínculo familiar ou Que não dispõem de condições para prover sua própria subsistência. As Instituições de Longa Permanência para Idosos (ILPIs), conhecidas também como asilos, abrigos, lares, casas de repouso, clínicas geriátricas e ancionatos, devem satisfazer às necessidades desses idosos Quanto à moradia, alimentação, saúde e convivência social, por meio do trabalho da assistência social, medicina, psicologia, enfermagem, fisioterapia, terapia ocupacional, odontologia, nutrição, entre outros serviços ${ }^{(7)}$.

A institucionalização do idoso deve ser a última alternativa de assistência ${ }^{(8)}$, pois representa um fator de risco para Quedas, uma vez Que a mudança do ambiente familiar para um ambiente estranho pode predispor a alterações psicológicas, cognitivas e funcionais relacionadas ao isolamento, abandono e inatividade física do indivíduo, acarretando em aumento da dependência para realização das atividades de vida diária e conseQuente redução da capacidade funcional ${ }^{(5)}$.

Desta forma, o declínio funcional torna o idoso institucionalizado mais suscetível a novas Quedas, comprometendo ainda mais sua independência. $\mathrm{O}$ delineamento deste contexto denota a relevância do assunto uma vez Que a Questão das Quedas deve ser tratada como um sério problema de saúde pública em razão de sua frequência, morbidade e do alto custo para os serviços de saúde $e^{(5)}$.

Sendo a incidência de Quedas um indicador de Qualidade da assistência de enfermagem ${ }^{(9)}$, é fundamental a implantação de protocolo de avaliação de risco para Quedas na população idosa, como instrumento revelador da realidade da instituição e norteador de intervenções de prevenção destes eventos.

Diante da importância desta problemática, desenvolvemos a presente pesQuisa com os seguintes objetivos: Verificar a prevalência de Quedas em idosos residentes em uma Instituição de Longa Permanência localizada no município de São Paulo; Caracterizar os idosos Que sofreram Quedas no período estudado, com relação a variáveis demográficas e clínicas; Descrever as características das Quedas ocorridas em relação a sua frequência, causas, circunstâncias e consequências.

\section{MÉTODO}

Trata-se de um estudo Quantitativo descritivo e retrospectivo, desenvolvido numa Instituição de Longa Permanência para Idosos, localizada no município de São Paulo. Esta instituição, existente há 34 anos, tem caráter filantrópico e é gerenciada pela Liga Solidária, entidade sem fins lucrativos Que gerencia outras unidades de atenção a crianças, jovens, adultos e idosos. A ILPI atende a idosos independentes e total ou parcialmente dependentes, Que, no entanto, não apresentam Quadro demencial grave. Conta com uma equipe multiprofissional composta por médicos, enfermeiras, auxiliares e técnicos de enfermagem, nutricionistas, fisioterapeutas, fonoaudiólogas, terapeuta ocupacional, psicóloga, cuidadores e voluntários.

A população do estudo foi composta por todos os 121 prontuários dos residentes da instituição, de sexo feminino e masculino, com idade igual ou superior a 60 anos. Levantamos registros de Quedas ocorridas entre agosto de 2006 e agosto de 2007, sendo a coleta de dados efetuada em fevereiro de 2008.

Para a coleta de dados, foi utilizado um instrumento semiestruturado elaborado a partir de pesQuisa bibliográfica, contendo informações sobre o idoso e a Queda. As variáveis sexo, idade, antecedentes clínicos pessoais, presença de déficit cognitivo, número de Quedas anteriores e medicações em uso, foram obtidas do prontuário do residente. Informações sobre o grau de dependência para Atividades de Vida Diária (AVD) e uso de equipamentos de ajuda na locomoção foram obtidos por relato das enfermeiras da instituição. As variáveis sobre a história da Queda (data, hora, local, condições do piso, posição inicial do idoso, situação de ocorrência da Queda e conseQuências físicas) foram conseguidas a partir do relatório específico da ILPI para notificação dos eventos. Esta ficha de notificação é preenchida imediatamente após a ocorrência da Queda, pelo funcionário Que encontrou o 
idoso, e contém uma breve avaliação do médico e/ou enfermeira de plantão e a conduta estabelecida por eles.

O estudo passou por autorização dos diretores da instituição por meio de uma solicitação escrita e aprovação do Comitê de Ética em Pesquisa da Universidade Federal de São Paulo sob protocolo número 2036/07.

Foi realizada uma análise descritiva das frequências obtidas. A discussão foi realizada à luz da literatura sobre o assunto.

\section{RESULTADOS}

Durante o período estudado, de um ano, foram analisados $12 \mathrm{I}$ prontuários, a partir dos Quais encontramos 87 relatórios de ocorrência de Quedas. Pelos documentos analisados, identificamos a ocorrência de 114 Quedas no período, sofridas por 45 idosos, gerando uma média de 2,53 $\pm 2,9$ Quedas por idoso. A prevalência encontrada no nosso estudo foi de $37,2 \%$.

\section{Caracterização dos idosos}

Do total de 45 idosos, $38(84,4 \%)$ eram do sexo feminino e sete $(15,6 \%)$ do sexo masculino. É uma característica da instituição a predominância de residentes do sexo feminino. A faixa etária mais prevalente foi de 80 a 85 anos, com $12(26,7 \%)$ idosos. A idade média foi de $83,75 \pm 14$ anos. A distribuição das faixas etárias é mostrada na Tabela 1.

Dentre os residentes que caíram, 2l (46,7\%) sofreram mais de uma Queda no período estudado, segundo mostra a Tabela 2.

Em nosso estudo, os residentes Que sofreram Quedas apresentaram diversos diagnósticos médicos. A hipertensão arterial sistêmica estava presente em mais da metade dos idosos Que caíram (53,3\%). Já as cardiopatias (insuficiência cardíaca, fibrilação atrial, insuficiência coronariana, infarto agudo do miocárdio, arritmia e taquicardia sinusal) foram encontradas em $46,6 \%$ dos idosos. Em três $(6,7 \%)$ prontuários não constavam registros dos diagnósticos

Tabela 1. Residentese suas faixas etárias. São Paulo, 2009.

\begin{tabular}{ccc}
\hline \multirow{2}{*}{ Faixas etárias } & \multicolumn{2}{c}{ Residentes } \\
\cline { 2 - 3 } & $\mathbf{n}$ & $\%$ \\
\hline 60 a 75 & - & 0,0 \\
76 a 80 & 7 & 15,6 \\
81 a 85 & 12 & 26,7 \\
86 a 90 & 11 & 24,4 \\
91 a 95 & 11 & 24,4 \\
> 95 & 3 & 6,7 \\
Não informado & 1 & 2,2 \\
\hline Total & $\mathbf{4 5}$ & $\mathbf{1 0 0 , 0}$ \\
\hline
\end{tabular}

Tabela 1. Número de quedas anteriores São Paulo, 2009.

\begin{tabular}{ccc}
\hline \multirow{2}{*}{ Quedas anteriores } & \multicolumn{2}{c}{ Residentes } \\
\cline { 2 - 3 } & $\mathbf{n}$ & $\%$ \\
\hline 0 & 24 & 53,3 \\
1 & 7 & 15,6 \\
2 & 7 & 15,6 \\
3 & 2 & 4,4 \\
4 ou mais & 5 & 11,1 \\
\hline Total & $\mathbf{4 5}$ & $\mathbf{1 0 0 , 0}$ \\
\hline
\end{tabular}

médicos dos residentes. A distribuição de diagnósticos encontrados é apresentada na Figura I.

Em decorrência da diversidade de doenças Que os idosos com Quedas apresentaram, o número de medicamentos prescritos também foi elevado. Mais da metade dos idosos $(60,0 \%)$ fazia uso de anti-hipertensivos tais como vasodilatadores, diuréticos e betabloqueadores. Os antidepressivos estavam prescritos para $53,3 \%$ dos idosos, os benzodiazepínicos para $17,8 \%$, os neurolépticos para $\mathrm{II}, \mathrm{I} \%$ e os hipoglicemiantes orais para $4,4 \%$. Nos prontuários de sete idosos $(15,6 \%)$ não havia registros da prescrição médica no período estudado. A quase totalidade da população $(80,0 \%)$ fazia uso de outros medicamentos, categoria Que compreende corticosteróides, anti-inflamatórios não-hormonais, miorrelaxantes, laxativos, protetores gástricos, anti-histamínicos, complexos vitamínicos e diversos outros.

Encontramos em 27 (60,0\%) idosos alguma alteração do estado cognitivo. Quanto ao grau de dependência para AVD, 25 idosos $(55,6 \%)$ eram parcialmente dependentes, $14(31,1 \%)$ independentes e seis $(13,3 \%)$ totalmente dependentes.

Dentre os equipamentos de ajuda na locomoção, a bengala foi a mais usada por 19 idosos $(42,2 \%)$. Dezesseis idosos $(35,6 \%)$ não precisavam de ajuda para deambular, oito $(17,8 \%)$ utilizavam cadeira de rodas e um $(2,2 \%)$ utilizava andador. A informação de Qual equipamento para locomoção os residentes utilizavam foi obtida por relato das enfermeiras, uma vez Que esta informação não estava registrada em todos os prontuários.

\section{Descrição das Quedas}

As Quedas se deram em todos os períodos do dia: $32,4 \%$ de manhã; $13,1 \%$ à tarde; $22,8 \%$ à noite; $12,3 \%$ durante a madrugada. Em 19,3\% não houve registro do horário da ocorrência.

A posição inicial dos idosos Que caíram foi predominantemente em pé, com 89 casos $(78,1 \%)$. Obtivemos 14 Quedas $(12,3 \%)$ a partir da posição sentada e três $(2,6 \%)$ no nível deitado. Em oito $(7,0 \%)$ casos não havia registro da posição inicial dos idosos. A condição do piso foi a variável mais defasada em termos de resposta. Em 96 eventos $(84,2 \%)$ não foram registradas as condições do piso. Não houve registros descrevendo Quedas em piso seco. Em nove casos $(7,9 \%)$ o piso estava molhado, em Quatro $(3,5 \%)$ havia obstáculos (poltrona, tapete, calçada irregular), três $(2,6 \%)$ ocorreram em rampas e dois $(1,8 \%)$ em degraus.

As situações no momento do evento foram diversas. Em 50 eventos $(43,9 \%)$ os residentes estavam deambulando; em II,4\% estavam se levantando da cama, sofá ou cadeira; em 1,7\% estavam se sentando; e em $0,9 \%$, se abaixando. Outras situações ocorreram em 14,9\% dos casos, incluindo síncope, tontura, hipotensão, malestar, desequilíbrio, rolamento da cama e Quebra de cadeira. Nenhum indivíduo, em nosso estudo, estava buscando objeto elevado, subindo ou descendo escada. Em 31 Quedas $(27,2 \%)$ não encontramos registro da situação de ocorrência do evento.

O local de maior ocorrência de Quedas foi o quarto do idoso, com 49 eventos $(43,0 \%)$, seguido pelo banheiro com $21,0 \%$, corredor com $8,8 \%$, e outros locais com $4,4 \%$ (elevador, portaria, recepção e sala de fisioterapia). Ainda ocorreram Quatro Quedas $(3,5 \%)$ no refeitório, três $(2,5 \%)$ Quedas fora da ILPI, duas $(1,8 \%)$ no jardim e uma $(0,9 \%)$ na sala de estar. Em 16 eventos $(14,0 \%)$ não foram registrados o local de ocorrência. 


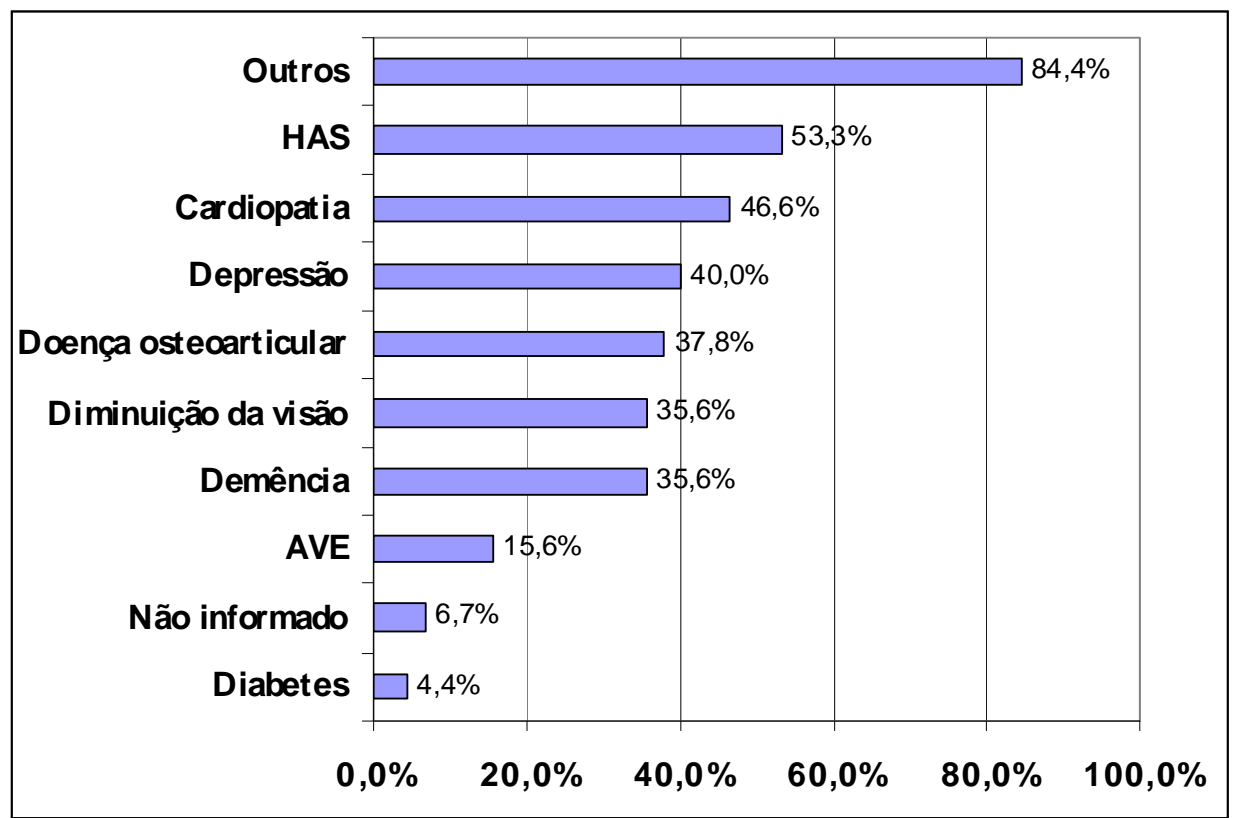

Figura 1. Diagnósticos dos pacientes que sofreram quedas. São Paulo, 2009.

acontecimento das Quedas, como declínio da força muscular, alterações na massa óssea, déficit de equilißbrio ${ }^{(10)}$, lentificação do tempo de reação, aumento do balanço do corpo, declínio dos reflexos, redução do controle postural ${ }^{(17)}$, da coordenação motora, da flexibilidade, além de deficiências de visão, propriocepção, vibração e do sistema vestibular ${ }^{(12)}$.

Mais de dois terços dos idosos Que sofrem uma Queda cairão novamente nos seis meses subsequentes $^{(16)}$. Isto significa Que história de Queda anterior, em pelo menos seis meses passados, é um fator preditor de uma nova Queda ${ }^{(18)}$.

Os fatores relacionados às Quedas de idosos são múltiplos e comumente classificados em intrínsecos e extrínsecos ao individuo. Os fatores intrínsecos

Em 57,3\% das Quedas houve alguma consequência física para o idoso. Ao todo, foram registradas 81 lesões em 66 Quedas.

Não encontramos registro de perda de consciência no local do evento, nem de óbitos em até um mês após Que poderiam estar relacionados à Queda. Apesar do número significante de lesões, apenas em 12 ocorrências (10,5\%) houve a necessidade de avaliação clínica hospitalar, com seis $(5,3 \%)$ delas resultando em internação.

Em 7,0\% das Quedas, os idosos sofreram posterior limitação para a realização de AVD. Este valor pode estar subestimado pela deficiência dos registros de avaliação do grau de dependência dos idosos.

\section{DISCUSSÃO}

A prevalência de Quedas encontrada no nosso estudo foi de $37,2 \%$. Este valor é comparável ao encontrado em estudos sobre Quedas de idosos residentes em comunidade: $34,8 \%^{(10)}$ e $30,9 \%$ (III). Em pesquisa com idosos institucionalizados na Bahia, encontrouse uma prevalência de $29,4 \%{ }^{(12)}$. Cerca de $50 \%$ de residentes em ILPIs sofrem pelo menos uma Queda no período de um ano ${ }^{(13)}$.

Inúmeros estudos têm encontrado uma frequência de Quedas maior em mulheres do Que em homens ${ }^{(11-12,14-15)}$. Ainda não há uma explicação conclusiva sobre este fato, o Qual parece estar relacionado ao melhor estado funcional das mulheres idosas em comparação aos homens e maior mobilidade com consequente maior exposição ao risco de Queda ${ }^{(14)}$. Também há maior fragilidade entre as mulheres, pois a euantidade de massa magra e a força muscular são menores em idosas ${ }^{(I I)}$. Outros autores também citam a utilização de maior Quantidade de drogas ${ }^{(15)} \mathrm{e}$ alta prevalência de doenças crônicas entre as mulheres ${ }^{(12)}$.

A literatura tem demonstrado Que a incidência dos eventos aumenta com o avançar da idade ${ }^{(1,12-15)}$. Entre 65 e 74 anos a taxa de quedas é de 32\%; entre 75 e 84 anos, 35\%; e acima de 85 anos, $51 \%{ }^{(16)}$. O envelhecimento acarreta alterações fisiológicas Que favorecem o incluem as diversas patologias, alterações fisiológicas do envelhecimento e consumo de medicamentos ${ }^{(19)}$. Dentre as patologias Que predispõe a Quedas, as mais comuns são as cardiovasculares, neurológicas, endócrino-metabólicas, osteomusculares, psieuiátricas, geniturinárias, pulmonares e sensoriais ${ }^{(15-16,19)}$. A hipertensão arterial esteve presente em $53,3 \%$ dos idosos Que caíram. Ainda existe controvérsia na literatura científica sobre associação entre hipertensão e Quedas, uma vez Que alguns estudos não encontraram esta associação, enQuanto Que outros a relacionam a hipotensão postural causada pelo uso de anti-hipertensivos ${ }^{(13,17)}$.

O diagnóstico de cardiopatia esteve presente em $46,6 \%$ dos gerontes. A Queda pode ser o primeiro sinal de um infarto agudo do miocárdio assintomático. Certas arritmias como os bloqueios atrioventriculares, alterações do nó sinusal e bradicardias também podem ocasionar Quedas ${ }^{(1)}$.

A depressão, presente em $40,0 \%$ dos residentes, é uma doença frequentemente relacionada a Quedas ${ }^{(13,16,18)}$. A presença de sintomas depressivos é um importante fator preditor para duas ou mais Quedas, em decorrência do efeito de medicações antidepressivas e sedativas, do declínio da capacidade funcional, baixa autoconfiança, indiferença ao meio ambiente, reclusão e inatividade ${ }^{(20)}$.

A demência e a diminuição da acuidade visual estiveram presentes em 16 idosos (35,6\%). A presença de demência pode aumentar o número de Quedas por conta de alterações da percepção espacial e habilidade de se orientar geograficamente ${ }^{(21)}$. Déficit visual é considerado, por outros autores, um importante fator de risco para esses eventos ${ }^{(13,17,22)}$.

O uso de medicamentos é um fator intrínseco de forte relação com as Quedas. Em função da diversidade de doenças apresentadas pelos idosos, é comum encontrarmos a polifarmácia entre os residentes, o Que é preocupante, uma vez que os efeitos da interação medicamentosa são mais acentuados nos idosos devido às alterações na absorção, metabolismo e eliminação das drogas Que decorrem do envelhecimento ${ }^{(23)}$. 
O risco de Quedas é aumentado pelo uso de drogas cardiovasculares (drogas de maior uso pela população estudada), pois produzem hipotensão, bradicardia, sonolência e fadiga ${ }^{(24)}$. Os diuréticos promovem depleção de volume e distúrbios hidroeletrolíticos ${ }^{(19)}$, embora possa haver um efeito protetor devido à redução na excreção urinária de cálcio e conseQuente aumento da densidade óssea ${ }^{(25)}$. Os antidepressivos e benzodiazepínicos podem causar sedação, alterações psicomotoras, relaxamento muscular e bloqueio beta-adrenérgico, aumentando a ocorrência de hipotensão ortostática $^{(24-25)}$, Que por sua vez aumenta a propensão a Quedas.

Em nosso estudo, 60,0\% dos idosos apresentavam algum prejuízo cognitivo. Alguns autores têm observado relação entre alterações cognitivas e Quedas ${ }^{(11,13-14,21-22)}$. Idosos com declínio cognitivo têm cinco vezes mais chances de sofrer Quedas ${ }^{(12)}$. As principais funções cognitivas Que contribuem para o controle postural e manutenção do eQuilíbrio são a atenção, memória e orientaçã̃o ${ }^{(11-12,18)} \mathrm{e}$, Quando comprometidas, também prejudicam a adaptação ao ambiente, julgamento e adoção de atitudes Que não lhe ofereçam risco ${ }^{(12)}$.

Verificamos que a maioria dos idosos que caíram é parcialmente dependente. Sabe-se Que a redução na capacidade de realização das atividades de vida diária é um importante fator de risco ${ }^{\text {(II, I- }}$ 14,17-18,21-22). Em uma ILPI na Alemanha, os residentes moderadamente dependentes tiveram uma incidência de quedas maior do Que os independentes ou gravemente dependentes ${ }^{(18)}$. Além dos problemas com equilíbrio, marcha, mobilidade e força muscular, o idoso com declínio funcional apresenta risco aumentado para Quedas em decorrência da baixa autoconfiança para realizar as AVD, o Que compromete ainda mais sua capacidade funcional ${ }^{(I I)}$. Por isso, o grupo dos parcialmente dependentes carece de mais atenção, visto Que possui um declínio funcional significante, porém não determinante para completa imobilidade, em comparação ao grupo dos totalmente dependentes. De maneira Que a Queda entre os residentes do último grupo pode representar, de certa forma, uma iatrogenia da equipe de enfermagem e de cuidadores.

Observamos, ainda, Que a avaliação da capacidade funcional dos residentes, na instituição estudada, não é feita por instrumentos como a Avaliação de Kenny para os Cuidados Pessoais ou o Índice de KATZ para Atividades de Vida Diária ${ }^{(26)}$. Esta característica é verificada subjetivamente pelas enfermeiras conforme necessidade de auxílio do residente para higiene, alimentação e deambulação.

Em relação à marcha, vimos Que $42,2 \%$ dos idosos utilizam bengala e 35,6\% não necessitam de ajuda pra deambular. O idoso com marcha livre ou Que utiliza bengala possui maior liberdade de locomoção e sensação de segurança, em comparação ao idoso Que faz uso de andador ou cadeira de rodas, fazendo com Que se exponha mais e caia mais vezes ${ }^{(12,21)}$. O fato de os idosos com bengalas sofrerem mais Quedas, no nosso estudo, indica a necessidade de treinamento para a utilização desse tipo de órtese como estratégia de redução da incidência desses eventos.

Obtivemos maior índice de Quedas pela manhã, dado semelhante ao encontrado em outra pesquisa ${ }^{(12)}$. As Quedas ocorrem nesse período, pois é o horário de maior atividade dos idosos. Esta informação é importante para o planejamento de cuidados de enfermagem, alertando a equipe para a prevenção de Quedas neste horário.

Assim como em nossa investigação, a maioria dos idosos caem da própria altura ${ }^{(15)}$. Os principais problemas com o ambiente, segundo a literatura, são piso escorregadio, objetos no chão, buscar objetos elevados, rolar da cama e problemas com degraus ${ }^{(15,27)}$. A descrição da cena do evento é um dado importante para implantação de medidas preventivas em razão da íntima relação entre ambiente e Quedas, pois sabe-se Que os perigos ambientais são causa de Quedas em $16 \%$ dos eventos em ILPIs e $41 \%$ em comunidade ${ }^{(16,21)}$.

Os dados sobre as circunstâncias dos eventos foram semelhantes aos encontrados em outra pesquisa ${ }^{(14)}$, onde a maioria dos idosos estava deambulando Quando caiu. Estudos têm mostrado Que grande parte das Quedas em ILPIs ocorrem durante transferências para cama, cadeira ou cadeira de rodas ${ }^{(18,21)}$. A informação sobre a circunstância da Queda pode direcionar a avaliação médica para a causa do evento, por exemplo: cair após se levantar de uma posição mais baixa pode estar relacionado à hipotensão postural; Quedas após tropeço ou escorregão indicam presença de fator ambiental ou problemas de marcha, equilíbrio e visão ${ }^{(22)}$.

As Quedas em ILPIs e em hospitais ocorrem principalmente nos Quartos e banheiros ${ }^{(19)}$, por estes serem os espaços mais utilizados pelos idosos. Conhecer o local de maior prevalência de Quedas numa instituição é fundamental para a priorização de cuidados preventivos específicos de cada ambiente.

Apesar de a maioria dos eventos registrados não resultarem em lesões, sabemos Que cerca de $40 \%$ a $60 \%$ das Quedas levam a ferimentos, sendo $30 \%$ a $50 \%$ de ferimentos leves, $5 \%$ a $6 \%$ de lesões mais graves excluindo fraturas e $5 \%$ de fraturas ${ }^{(1,16,2,21)}$. Em nosso estudo, observamos Que $11,4 \%$ de lesões ocorreram na região da cabeça, o Que poderia resultar em graves lesões cerebrais com prejuízo às funções vitais e risco à vida.

Com respeito às fraturas, a literatura demonstra Que as mais comuns são as de Quadril $(25 \%)^{(1)}$. Este tipo de fratura é mais prevalente em indivíduos acima de 75 anos, decorrente, possivelmente, da lentificação dos reflexos e do prejuízo na habilidade de proteger o Quadril durante a Queda se apoiando sobre o punho ${ }^{(22)}$. Cerca de $25 \%$ dos idosos com este tipo de fratura morrem após seis meses do evento e $60 \%$ apresentam incapacidade de realizar suas funções habituais ${ }^{(19)}$.

As Quedas em ILPIs tendem a resultar em consequências mais sérias do Que as em comunidade, de modo Que a cada ano 1.800 eventos fatais ocorrem em instituições ${ }^{(2)}$. Entre os idosos com 85 anos ou mais, uma em cada cinco Quedas em ILPIs é fatal ${ }^{(19,21)}$. Não encontramos óbitos em até um mês do evento.

A instituição estudada oferece atendimento médico e de enfermagem em tempo integral, por isso os residentes que caíram foram avaliados e somente encaminhados a hospitais Quando o profissional julgava necessária a realização de exames específicos, o Que justifica o número relativamente baixo de encaminhamentos. Cerca de 7,0\% das pessoas de mais de 75 anos Que sofrem Quedas buscam atendimento em serviços de emergência, e 40,0\% destas necessitam de internação hospitalar ${ }^{(19)}$.

Em 7,0\% das Quedas, os idosos sofreram limitação para realização de AVD. A perda da independência pode ser consequência direta de lesões causadas pelo impacto ou consequência indireta pelo medo de voltar a cair (síndrome pósQueda). Trata-se de uma limitação funcional autoimposta causada pela perda da confiança em deambular sozinho com segurança. Além do declínio funcional, o idoso pode desenvolver depressão, 
sentimento de impotência e isolamento social ${ }^{(21)}$. Desta forma, ao deixar de realizar suas atividades rotineiras o idoso pode desenvolver a síndrome da imobilidade e, assim, aumentar o risco de vir a ter novas Quedas ${ }^{(15,22)}$.

\section{CONCLUSÃO}

Os resultados encontrados demonstram que a prevalência de Quedas em idosos institucionalizados é elevada, sendo mais frequente em mulheres de idade avançada, as Quais sofrem Quedas recorrentes, são portadoras de diversas patologias, fazendo uso de vários medicamentos, apresentam algum grau de déficit cognitivo e funcional e locomovem-se com auxílio de bengala. Com relação às Quedas, estas se deram em sua maioria pela manhã, da própria altura do idoso, enquanto ele deambulava no seu próprio Quarto, gerando algum tipo de conseQuência física para o idoso, sendo a mais comum os hematomas/sangramentos.

Embora nossa investigação tenha um caráter descritivo e não procure realizar inferências de causa e efeito entre as variáveis nem tencione levantar fatores de risco, foi possível observar que nossos resultados são coerentes com o encontrado na literatura científica sobre o assunto, mesmo Que alguns dos nossos dados sejam comparáveis aos de estudos com idosos residentes em comunidade.

No entanto, algumas limitações do estudo precisam ser consideradas, principalmente em relação ao levantamento de dados, o Qual foi realizado retrospectivamente a partir dos registros sobre os eventos, sem um aviso anterior dos funcionários Que realizaram tais notificações. Seria interessante realizar um estudo prospectivo a partir dos relatos imediatos das Quedas, com treinamento prévio dos funcionários Que irão anotar as ocorrências, no sentido de evitar tantas perdas por falhas de registro.

Notamos Que as Quedas em idosos são fatos comuns, inclusive dentro de instituições de longa permanência, com consequências significantes à saúde física, psicológica e social. É importante uma avaliação constante dos profissionais de saúde sobre os idosos caidores e não-caidores, no sentido de identificar os fatores de risco intrínsecos e extrínsecos para implementar estratégias de prevenção Que compreendam reabilitação da força muscular, equilibrio e capacidade funcional, redução da polifarmácia, educação para o autocuidado e aumento da supervisão de enfermagem nos períodos e locais de maior incidência de Quedas, melhorando desta forma a Qualidade de vida dos seus residentes.

\section{Agradecimentos}

Destacamos nossos agradecimentos à diretoria da ILPI pelo interesse e autorização para execução da pesquisa, e à equipe de enfermagem pela recepção e colaboração às pesquisadoras.

\section{REFERÊNCIAS}

I. Ministério da Saúde (BR). Secretaria de Atenção à Saúde. Departamento de Atenção Básica. Envelhecimento e saúde da pessoa idosa. Brasília: Ministério da Saúde, 2006. [citado em: 2007 Jul 27]. Disponível em: http://dtr200l.saude.gov.br/editora/ produtos/livros/popupenvelhecimento_saude_pessoa_idosa.html

2. São Paulo (SP). Secretaria Municipal de Assistência e Desenvolvimento Social. Cartilha do Idoso: mitos e verdades sobre a velhice: guia de serviços. São Paulo: SMADS; 2006.

3. Brasil. Portaria $\mathrm{n}^{\circ}$ 2.528, de 19 de outubro de 2006. Aprova a Política Nacional de Saúde da Pessoa Idosa. Diário Oficial União 2006 out 20; I: 142-5.

4. Duarte YAO. Indicadores de fragilidade em pessoas idosas visando o estabelecimento de medidas preventivas. Bol Inst Saúde 2009; (47): 49-52.

5. Perracini MR. Prevenção e manejo de Quedas. In: Ramos LR coordenação. Guia de geriatria e gerontologia. Barueri: Manole; 2005. p.193-208.

6. Ministério da Saúde (BR). DATSUS. Informações de saúde. Óbitos por causas externas. Brasília: Ministério da Saúde, 2008. [citado em 2009 Set II]. Disponível em: http:// tabnet.datasus.gov.br/cgi/tabcgi.exe?simp/cnv/extuf.def

7. Sociedade Brasileira de Geriatria e Gerontologia. Seção São Paulo. Instituição de longa permanência para idosos: manual de funcionamento. São Paulo: SBGG; 2003.

8. Ministério da Saúde (BR). Estatuto do Idoso. Brasília: Ministério da Saúde; 2003.

9. Programa de Qualidade Hospitalar. Manual de Indicadores de Enfermagem NAGEH. São Paulo: APM/CREMESP, 2006.

10. Siqueira FV, Facchini LA, Piccini RX, Tomasi E, Thumé E, Silveira DS, et al. Prevalência de Quedas em idosos e fatores associados.
Rev Saúde Pública 2007; 4I(5): 749-56.

I I . Perracini MR, Ramos LR. Fatores associados a Quedas em uma coorte de idosos residentes na comunidade. Rev Saúde Pública 2002; 36(6): 709-16.

12. Santos MLC, Andrade MC. Incidência de Quedas relacionada aos fatores de risco em idosos institucionalizados. Rev Baiana de Saúde Pública 2005; 29: 57-68.

13. Masud T, Morris RO. Epidemiology of falls. Age Aging 2001; 30-S4: 3-7.

14. Gac H, Marin PP, Castro S, Hoyl T, Valenzuela E. Caídas em adultos mayores institucionalizados: descripción y evaluación geriátrica. Rev Méd Chile 2003; I3I: 887-94.

15. Fabrício SCC, Rodrigues RAP e Costa Junior ML. Causas e consequências de Quedas de idosos atendidos em hospital público. Rev Saúde Pública 2004; 38(I): 93-9.

16. Pereira SRM, Buksman S, Perracini M, Py L, Barreto KML, Leite VMM. Sociedade Brasileira de Geriatria e Gerontologia. Projeto Diretrizes. Quedas em idosos. São Paulo: Associação Médica Brasileira, Conselho Federal de Medicina; 2001.

17. Chan KM, Pang WS, Ee CH, Ding YY, Choo P. Epidemiology of falls among the elderly community dwellers in Singapore. Singapore Med I 1997; 38(10): 427-31.

18. Kron M, Loy S, Sturm E, Nikolaus Th, Becker C. Risk indicators for falls in institucionalized frail elderly. Am I Epidemiol 2003; 158: 645-53.

19. Brito FC, Costa SMN. Quedas. In: Papaleo Netto M, Brito FC. Urgências em geriatria. São Paulo: Atheneu; 200I. p. 32335 .

20. Whooley MA, Kip KE, Cauley IÁ, Ensrud KE, Nevitt MC, Browner WS. Depression, falls, and risk of fracture in older 
woman. Arch Intern Med 1999; 159(5): 484-90.

21. Rubenstein LZ, Josephson KR, Robbins AS. Falls in the nursing home. Ann Intern Med 1994; I2I(6): 442-51.

22. Rubenstein LZ. Falls in older people: epidemiology, risk factors and strategies for prevention. Age Ageing 2006; 35-S2: ii37ii4l.

23. Secoli SR. Polifarmácia: interações e reações adversas no uso de medicamentos por idosos. Rev Bras Enferm 2010; 63(I): 136-40.

24. Guimarães IMN, Farinatti PTV. Análise descritiva de variáveis teoricamente associadas ao risco de Quedas em mulheres idosas.
Rev Bras Med Esporte 2005; II(5): 299-305.

25. Coutinho ESF, Silva SD. Uso de medicamentos como fator de risco para fratura grave decorrente de Quedas em idosos. Cad Saúde Pública 2002; 18(5): 1359-66.

26. Ribeiro RC, Marin HE. Proposta de um instrumento de avaliação da saúde do idoso institucionalizado baseado no conceito do Conjunto de Dados Essenciais em Enfermagem. Rev Bras Enferm 2009; 62(2): 204-12.

27. Marin MIS, Amaral FS, Martin IB, Bertassi VC. Identificando os fatores relacionados ao diagnóstico de enfermagem "risco de Quedas" entre idosos. Rev Bras Enferm 2004; 57(5): 560-4. 